\title{
A Case of Therapy-Resistant Folliculitis due to Adrenal Insufficiency?
}

\author{
Kirsten Rønholt Stausholm ${ }^{a} \quad$ Eva Spaun $^{b} \quad$ Uffe Koppelhus ${ }^{a}$ \\ ${ }^{a}$ Department of Dermatology, Aarhus University Hospital, Aarhus, Denmark; \\ ${ }^{b}$ Department of Pathology, Aarhus University Hospital, Aarhus, Denmark
}

\section{Keywords}

Therapy-resistant folliculitis · Pathogenesis of folliculitis · Adrenal insufficiency

\section{Abstract}

A report on a 40-year-old patient with recalcitrant, suppurative folliculitis is presented. After years of unsuccessful treatment with conventional therapies, the patient was diagnosed with adrenal insufficiency with a low level of circulating cortisol. A few weeks after the patient was subjected to substitution therapy with hydrocortisone, his folliculitis resolved. We discuss the role of plasma cortisol level in the pathogenesis of folliculitis.

\section{Introduction}

The etiology of folliculitis is complex and in a given case several factors may contribute including infection, physical irritation, occlusion, other skin diseases and drugs. Among drugs, especially systemic and potent topical glucocorticoid treatment can cause acneiform eruptions [1]. However, the role of the native glucocorticoid, cortisol, as a regulator/ dampener of potential inflammatory reactions in the hair follicles is unknown.

Here we report a case of treatment-resistant folliculitis which resolved after recognition and treatment of low cortisol due to adrenal insufficiency. 


\section{Case Report}

A 40-year-old man was admitted to our clinic with folliculitis which he had suffered from for at least 2 years. Besides genital condyloma, he had never suffered from any skin diseases. He had a medical history of psychological problems, tiredness, low libido and lower back pain. As a supplement to body building, he had used anabolic steroids a short period of time more than 15 years before the debut of folliculitis.

Three years before the debut of folliculitis, he was involved in a traffic injury, accelerating all his symptoms. This led to an abuse of cocaine and amphetamine for 4 years, whereafter he was diagnosed with attention deficit and hyperactivity disorder and treated with methylphenidate. At this time, he was admitted to our clinic. He was continuously treated with morphine for his lower back pain.

Clinically, he presented a superficial folliculitis with inflamed red hair follicles with surface pustules. The skin changes were found on the scalp, face, upper trunk, thighs and pubic area. At first, the patient was treated with long-term courses (up to 6 months) of oral tetracycline supplemented with regimes of topical antifungals (ketoconazole shampoo 2\%) and, on separate occasions, short regimes of topical glucocorticoid (hydrocortisone-17-butyrate and betamethasone with clioquinol, respectively). All initiated treatments were found to be remarkably insufficient in this patient with little or no effect on clinical findings. A skin biopsy from the back of the upper trunk was performed which showed suppurative folliculitis, primarily involving the infundibular portions of the hair follicle. Periodic acid-Schiff stain revealed numerous oval yeast-like organisms, thus compatible with Malassezia Furfur folliculitis (fig. 1, fig. 2). Systemic antifungal treatment included itraconazole $100 \mathrm{mg}$ per day for 2 weeks with no clinical response. Treatment with fluconazole, at first $150 \mathrm{mg}$ every week for 8 weeks, followed by $300 \mathrm{mg}$ every week for 8 weeks, showed some response, but with persisting folliculitis in the beard area. Courses of systemic glucocorticoids given by the patient's general practitioner allegedly showed good effect. However, such treatment was tapered or discontinued at the follow-ups in our clinic.

Two years after admission to our clinic, the patient was admitted to the Department of Endocrinology, Odense University Hospital, Odense, Denmark. Blood tests showed low testosterone $(3.8 \mathrm{nmol} / \mathrm{l})$, low testosterone/sex hormone-binding globulin (0.09) and low luteinizing hormone (0.6 UI/l) compatible with hypogonadotropic hypogonadism. Also the cortisol level was low (spot cortisol $74 \mathrm{nmol} / \mathrm{l}$ ), with adrenocorticotropic hormone being low in the normal reference interval. Magnetic resonance imaging showed a normal pituitary gland. The adrenocorticotropic hormone test was compatible with secondary adrenal insufficiency. Several factors were suspected to have caused suppression of the pituitary gland (use of amphetamine/cocaine, anabolic steroids, traffic injury, and use of morphine) and therefore the exact time of onset could not be said. At first, he was given testosterone for 1.5 months without any observable effect on his skin symptoms. However, when he received both testosterone and hydrocortisone his folliculitis resolved completely in less than a month, and still after 2 years there has been no severe relapse (fig. 3 ).

\section{Discussion}

Pituitary insufficiency can cause isolated or multiple affection of hormonal production. In this patient, both the level of testosterone and cortisol was low. Normalizing the level of testosterone did not have any effect on his skin, but shortly after hydrocortisone substitution 
his folliculitis resolved. We hypothesize that low plasma cortisol triggered or worsened the patient's folliculitis.

It is well known that acneiform eruptions can be side effects to both systemic and strong topical glucocorticoid treatment. Systemic glucocorticoid suppresses the hypothalamuspituitary-adrenal (HPA) axis resulting in a reduction in endogenous cortisol production and thereby low circulating cortisol level. Topical glucocorticoid also suppresses the HPA axis, although at a physiological level, and only pathologically when exceeding accepted safety guidelines [2]. To our knowledge, it is unknown whether the low level of endogenous cortisol plays a role in the pathogenesis of acneiform eruptions during glucocorticoid treatment besides the possible direct effect of the glucocorticoid drug.

Studies on the direct effect of topical glucocorticoids on the development of acneiform eruptions suggest that acne is provoked by inducing rupture of the follicle where sebum seeps into the dermis [3]. Also keratinization and suppuration of follicular epithelium has been suggested as pathogenesis of glucocorticoid-induced acneiform eruptions [4]. However, these studies are few and it remains unclear whether the previously described physiological suppression of the HPA axis is involved in the pathogenesis of topical glucocorticoidinduced acneiform eruptions.

\section{Conclusion}

In conclusion, this case report suggests that low levels of cortisol may play a role as modifier of the inflammatory component of folliculitis and thus the pathogenesis of at least certain types of folliculitis.

\section{Statement of Ethics}

Informed consent for publishing the case was obtained from the patient.

\section{Disclosure Statement}

The authors report no conflicts of interest. There was no funding of this work.

\section{References}

1 Luelmo-Aguilar J, Santandreu MS: Folliculitis: recognition and management. Am J Clin Dermatol 2004;5:301-310.

2 Levin E, Gupta R, Butler D, Chiang C, Koo JY: Topical steroid risk analysis: differentiating between physiologic and pathologic adrenal suppression. J Dermatolog Treat 2014;25:501-506.

-3 Kaidbey KH, Kligman AM: The pathogenesis of topical steroid acne. J Invest Dermatol 1974;62:31-36.

4 Lobo A: Pathogenesis of drug induced acneiform eruptions. Indian J Dermatol Venereol Leprol 1992;58:159-163. 


\section{Case Reports in \\ Dermatology}

Case Rep Dermatol 2016;8:267-271

Stausholm et al.: A Case of Therapy-Resistant Folliculitis due to Adrenal Insufficiency?

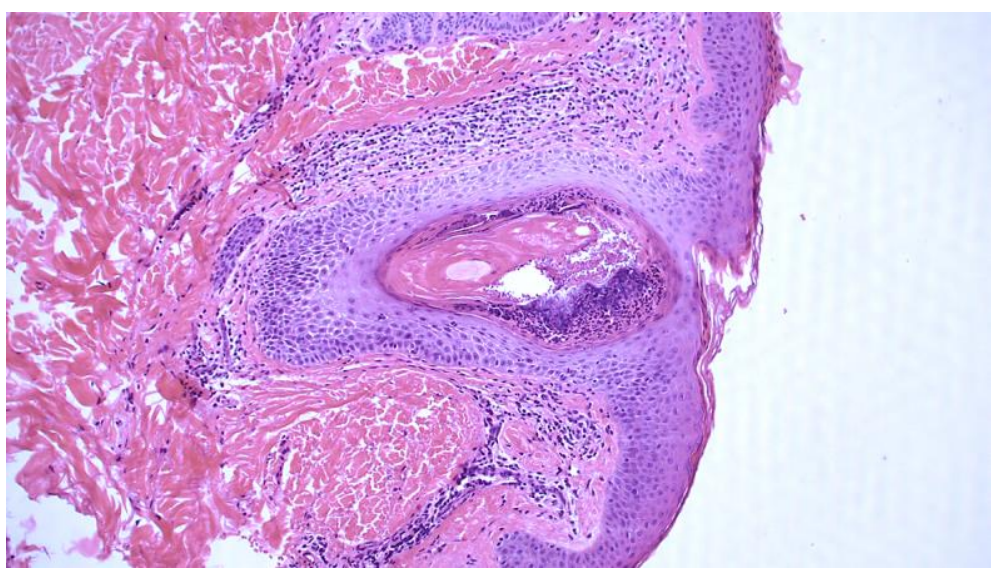

Fig. 1. Plugged hair follicle with infundibular dilatation and numerous polymorphonuclear leukocytes, cell debris and coccoid bacteria. There is a perifollicular lymphocytic infiltrate.

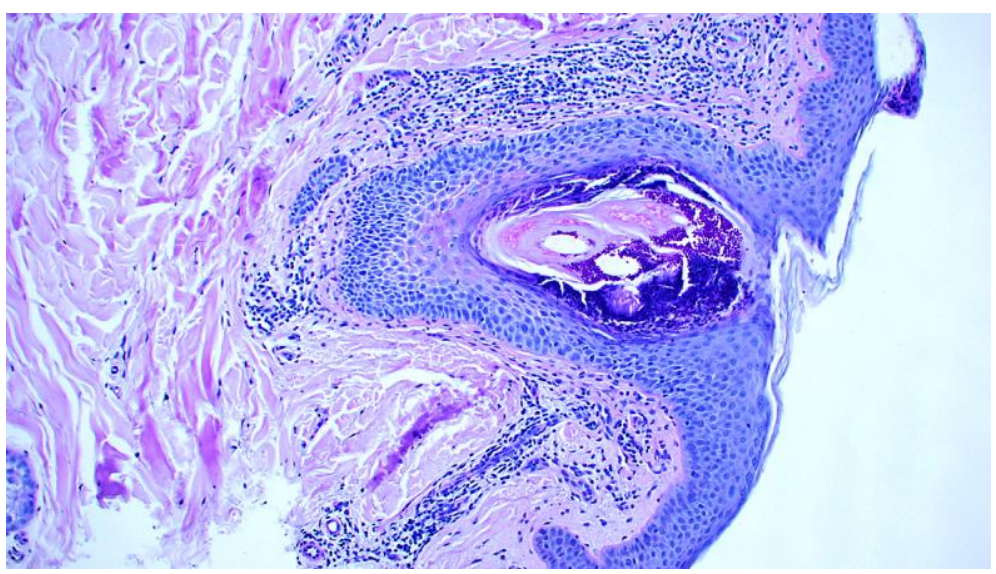

Fig. 2. Periodic acid-Schiff stain reveals numerous oval yeast-like organisms within the follicle. 


\section{Case Reports in Dermatology}

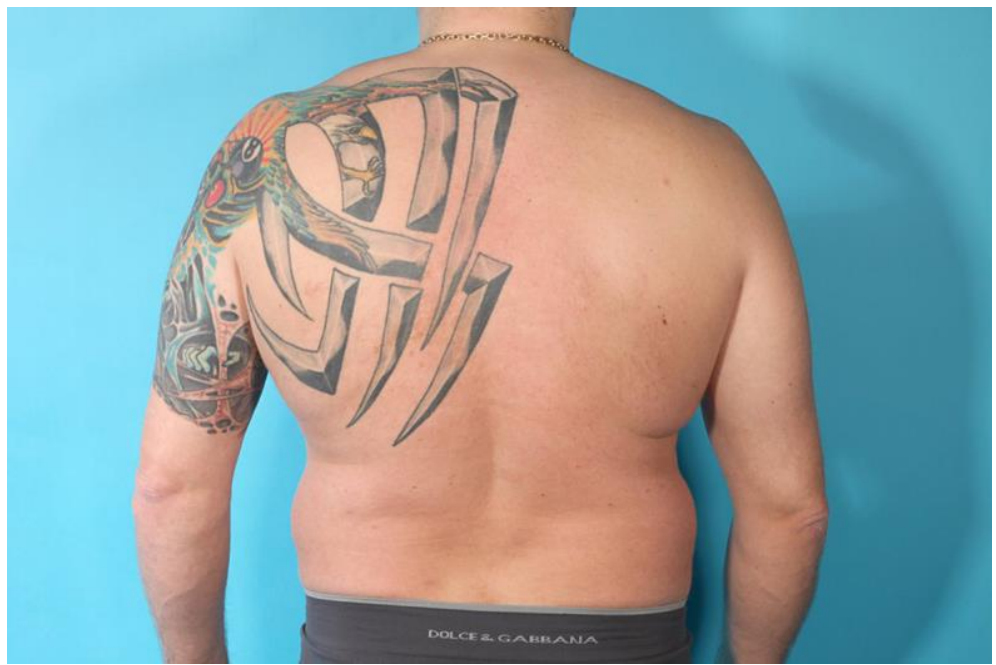

Fig. 3. Resent photo of the patient's back of the upper trunk illustrating no relapse of folliculitis without dermatological treatment. 